\title{
On tests for linearity against STAR models with deterministic trends*
}

\author{
Hendrik Kaufmann ${ }^{\ddagger}$, Robinson Kruse ${ }^{\S}$, Philipp Sibbertsen ${ }^{\dagger}$
}

\begin{abstract}
Linearity testing against smooth transition autoregressive (STAR) models when deterministic trends are potentially present in the data is considered in this paper. As opposed to recently reported results in Zhang (2012), we show that linearity tests against STAR models lead to useful results in this setting.
\end{abstract}

JEL-Codes: C12, C22

Keywords: Nonlinearity $\cdot$ Smooth transition · Deterministic trend

\footnotetext{
${ }^{*}$ We are grateful to an anonymous referee for helpful comments. Financial support by the Deutsche Forschungsgemeinschaft (DFG) is gratefully acknowledged. Robinson Kruse gratefully acknowledges financial support from CREATES funded by the Danish National Research Foundation.

$\ddagger$ Leibniz University Hannover, School of Economics and Management, Institute of Statistics, Königsworther Platz 1, D-30167 Hannover, Germany. E-Mail: kaufmann@statistik.unihannover.de. Phone: +49-511-762-5636.

$\S$ Leibniz University Hannover, School of Economics and Management, Institute of Statistics, Königsworther Platz 1, D-30167 Hannover, Germany. E-mail address: kruse@statistik.unihannover.de and CREATES, Aarhus University, School of Economics and Management, Bartholins Alle 10, DK-8000 Aarhus C, Denmark. E-mail address: rkruse@creates.au.dk .

${ }^{\dagger}$ Corresponding Author. Leibniz University Hannover, School of Economics and Management, Institute of Statistics, Königsworther Platz 1, D-30167 Hannover, Germany. E-Mail: sibbertsen@statistik. uni-hannover.de. Phone: +49-511-762-3783. Fax: +49-511-762-3923.
} 


\section{Introduction}

Nonlinear time series models like the smooth transition autoregressive (STAR) model, see Teräsvirta (1994), have become very popular during the last two decades. Testing for linearity is an elementary step in the modelling cycle and of great importance when it comes to building nonlinear models. Kiliç (2004) and Sandberg (2008) highlight certain difficulties for linearity tests when stochastic trends are present in the data, but neglected. Using standard critical values from the $\chi^{2}$-distribution can be misleading and results in size distortions. Amongst others, Harvey and Leybourne (2007) suggest a novel linearity test which is robust to the presence of stochastic trends, thereby providing an $\alpha$-level test when the time series under consideration is either stationary or nonstationary.

It is widely accepted that many economic and financial time series exhibit trending behavior. Analogous to neglecting stochastic trends, omission of deterministic trends can have severe consequences for linearity tests as well as shown in Zhang (2012). In order to cope with a linear deterministic trend, the author suggests OLS detrending prior to the application of a linearity test against nonlinear smooth transition autoregressive (STAR) models. The simulation results for such a procedure are discouraging due to very low power and Zhang (2012) concludes that "In most situations, this method is totally useless".

The aim of this article is to clarify some important issues and to re-consider linearity testing when a deterministic trend might be present in the data. First, we find that the poor results are mainly driven by the data generating process (DGP) which has been used in the Monte Carlo analysis of Zhang (2012). This DGP is partly problematic since (i) it does not ensure global stationarity and (ii) it includes a nonlinear trend. Obviously, it is difficult to remove a nonlinear trend by applying a linear detrending procedure. Even if the trend is linear, nonstationarity leads to a severe estimation bias of the intercept and the slope coefficient. Therefore, it is not surprising after all that the simulation results are indicating such a low power. When considering the DGP which has been used in Harvey and Leybourne (2007), we reverse the conclusion and find that the approach taken in Teräsvirta (1994) is actually useful in most situations. Furthermore, we suggest a slightly different approach and observe an even better performance in some situations. When comparing our results to an infeasible test which assumes a known trend function, we find only minor differences suggesting that our proposed method works well in finite samples. Our main conclusion is that linearity tests against STAR models are useful, 
even if linear deterministic trends are potentially present in the data. ${ }^{1}$ We conjecture that polynomial trends can be handled in a similar way. In a recent contribution, Cuestas and Garratt (2011) consider cubic trends in real GDP per capita series.

Section 2 covers different linearity tests, while Section 3 is dedicated to Monte Carlo simulations. Conclusions are drawn in Section 4.

\section{Testing methods}

We investigate LSTAR and ESTAR models with a linear deterministic trend:

$$
\begin{aligned}
& y_{t}=a+b t+v_{t} \\
& v_{t}=\rho v_{t-1}+\phi v_{t-1} F\left(v_{t-1} ; \gamma, c\right)+\varepsilon_{t},
\end{aligned}
$$

where $\varepsilon_{t}$ is a white noise process. Two standard smooth transition functions are considered:

$$
\begin{aligned}
& \text { ESTAR: } \quad F\left(v_{t-1} ; \gamma, c\right)=1-\exp \left[\gamma\left(v_{t-1}-c\right)^{2}\right] \\
& \text { LSTAR: } \quad F\left(v_{t-1} ; \gamma, c\right)=\frac{2}{1+\exp \left[-\gamma\left(v_{t-1}-c\right)\right]}-1 .
\end{aligned}
$$

This is a similar specification to the one in Harvey and Leybourne (2007) with an additional deterministic trend $b t$.

Zhang (2012) suggests the application of OLS detrending to $y_{t}$, i.e., $\tilde{y}_{t}=y_{t}-\tilde{\alpha}-\tilde{\beta} t$. Building upon the residuals $\tilde{y}_{t}$, Zhang (2012) uses the auxiliary regression

$$
\tilde{y}_{t}=\tilde{\beta}_{0}+\tilde{\beta}_{1} \tilde{y}_{t-1}+\tilde{\beta}_{2} \tilde{y}_{t-1}^{2}+\tilde{\beta}_{3} \tilde{y}_{t-1}^{3}+\tilde{\beta}_{4} \tilde{y}_{t-1}^{4}+\tilde{u}_{t}
$$

for both transition functions, see Teräsvirta (1994). The null hypothesis of linearity is $H_{0}: \tilde{\beta}_{2}=\tilde{\beta}_{3}=\tilde{\beta}_{4}=0$ with the Wald test statistic

$$
W=\frac{\mathrm{RSS}_{1}-\mathrm{RSS}_{0}}{\mathrm{RSS}_{0} / T},
$$

where $\mathrm{RSS}_{0}$ and $\mathrm{RSS}_{1}$ denote the residual sum of squares from the corresponding OLS regressions under $H_{0}$ and $H_{1}$ respectively.

Alternatively, we suggest a slightly different approach with an included trend in the auxiliary regressions denoted as TI in the following. The proposed test regressions are

${ }^{1}$ Our results do not depend on the actual presence of a linear trend in the data. 
based on Kiliç (2004) and given by

$$
\begin{array}{ll}
\text { ESTAR: } & y_{t}=\beta_{0}+\beta_{1} t+\beta_{2} y_{t-1}+\beta_{3} y_{t-1}^{2}+\beta_{4} y_{t-1}^{3}+u_{t}, \\
\text { LSTAR: } & y_{t}=\beta_{0}+\beta_{1} t+\beta_{2} y_{t-1}+\beta_{3} y_{t-1}^{2}+u_{t} .
\end{array}
$$

The null hypotheses of linearity are $H_{0}: \beta_{3}=\beta_{4}=0$ and $H_{0}: \beta_{3}=0$, respectively.

As a benchmark, we consider an infeasible test were the parameters $a$ and $b$ are both known. In this case, the linearity test is directly applied to $v_{t}=y_{t}-a-b t$ :

$$
\begin{array}{ll}
\text { ESTAR: } & v_{t}=\beta_{1} v_{t-1}+\beta_{2} v_{t-1}^{2}+\beta_{3} v_{t-1}^{3}+u_{t}, \\
\text { LSTAR : } & v_{t}=\beta_{1} v_{t-1}+\beta_{2} v_{t-1}^{2}+u_{t} .
\end{array}
$$

The null hypotheses of linearity are $H_{0}: \beta_{2}=\beta_{3}=0$ and $H_{0}: \beta_{2}=0$, respectively.

\section{Finite-sample properties}

The purpose of our Monte Carlo comparison is four-fold: (i) By using an appropriate data generating process (DGP) based on Harvey and Leybourne (2007), we investigate the power of linearity tests with deterministic trends, (ii) compare the approach taken in Zhang (2012) to our approach (TI), (iii) evaluate the relative performance with respect to the infeasible test, (iv) compare our results to those in Harvey and Leybourne (2007). In order to examine and compare the empirical power of the three different approaches outlined in Section 2, we consider the same parameterizations as in Harvey and Leybourne (2007) but with an additional linear deterministic trend. For our power experiment sample sizes of $T=150$ and $T=300$ are used with parameter settings $a=\{0,0.1\}$, $b=\{0,0.1\}, \rho=\{0,1,1.5\}, \phi=\{ \pm 0.5, \pm 0.7,-1,-1.4\}, \gamma=\{0.1,0.3,0.5,0.7,0.9\}, c=0$ and $\varepsilon_{t} \sim N(0,1)$. Only those parameter combinations are considered which ensure globally trend-stationary $I(0)$ processes. The results of the Monte Carlo simulation with 2,000 replications for $a=0$ and $b=0$ are shown in Table 1 and Table 3. Results for $a=0.1$ and $b=0.1$ are presented in Table 2 and 4 . All other parameterizations of $a$ and $b$ lead to very similar results as well and to the same conclusions. ${ }^{2}$

\footnotetext{
${ }^{2}$ Additional results for all other combinations of $a$ and $b$ are available in the online appendix, see http://www.statistik.uni-hannover.de/fileadmin/statistik/papers/STAR_trend_appendix.pdf . This appendix also contains additional detailed results on the empirical size of the different procedures. The main conclusion from these size experiments is that each test performs similarly well. The empirical size is usually close to the desired nominal $5 \%$ level significance.
} 
LSTAR

\begin{tabular}{rrrrrrrrrr}
\hline & & \multicolumn{3}{c}{$T=150$} & & \multicolumn{3}{c}{$T=300$} \\
\cline { 8 - 9 }$\rho$ & $\phi$ & $\gamma$ & Zhang & TI & Infeasible & & Zhang & TI & Infeasible \\
\hline 0 & 0.7 & 0.1 & 6.6 & 9.9 & 10.9 & & 9.2 & 13.2 & 16.9 \\
& 0.3 & 25.2 & 38.5 & 53.7 & & 49.7 & 67.2 & 84.0 \\
& 0.5 & 56.5 & 73.6 & 89.0 & & 89.4 & 94.9 & 99.5 \\
& 0.7 & 79.1 & 90.2 & 98.7 & & 98.6 & 99.8 & 100.0 \\
& 0.9 & 91.8 & 96.9 & 99.8 & & 99.6 & 100.0 & 100.0 \\
& 0.1 & 8.1 & 10.6 & 14.6 & & 12.4 & 18.5 & 26.4 \\
& 0.9 & 38.5 & 55.4 & 74.6 & & 73.1 & 87.2 & 96.5 \\
& 0.5 & 80.8 & 90.2 & 97.5 & & 98.9 & 99.9 & 100.0 \\
& 0.7 & 96.0 & 98.1 & 100.0 & & 100.0 & 100.0 & 100.0 \\
& 0.9 & 98.9 & 99.9 & 100.0 & & 100.0 & 100.0 & 100.0 \\
\hline
\end{tabular}

Table 1: Empirical power of different linearity tests at the nominal $5 \%$ significance level for $a=0$ and $b=0$. The DGP is given by equations in (1) and (3). Zhang is based on auxiliary regression (4), TI on (6) and Infeasible on (8).

LSTAR

\begin{tabular}{|c|c|c|c|c|c|c|c|c|}
\hline \multirow[b]{2}{*}{$\rho$} & \multirow[b]{2}{*}{$\phi$} & \multirow[b]{2}{*}{$\gamma$} & \multicolumn{3}{|c|}{$T=150$} & \multicolumn{3}{|c|}{$T=300$} \\
\hline & & & Zhang & $\mathrm{TI}$ & Infeasible & Zhang & $\mathrm{TI}$ & Infeasible \\
\hline \multirow[t]{10}{*}{0} & 0.7 & 0.1 & 7.1 & 9.0 & 9.8 & 8.0 & 12.5 & 17.6 \\
\hline & & 0.3 & 24.1 & 36.6 & 52.6 & 47.9 & 64.5 & 82.5 \\
\hline & & 0.5 & 59.3 & 74.5 & 90.0 & 90.0 & 96.0 & 99.6 \\
\hline & & 0.7 & 81.1 & 90.4 & 99.1 & 99.1 & 99.8 & 100.0 \\
\hline & & 0.9 & 92.2 & 97.2 & 99.9 & 99.9 & 100.0 & 100.0 \\
\hline & 0.9 & 0.1 & 8.8 & 12.2 & 14.6 & 11.6 & 18.6 & 24.3 \\
\hline & & 0.3 & 42.4 & 58.0 & 76.3 & 73.0 & 86.7 & 96.1 \\
\hline & & 0.5 & 79.9 & 89.9 & 98.2 & 98.4 & 99.8 & 100.0 \\
\hline & & 0.7 & 95.4 & 98.7 & 99.9 & 100.0 & 100.0 & 100.0 \\
\hline & & 0.9 & 99.2 & 99.7 & 100.0 & 100.0 & 100.0 & 100.0 \\
\hline
\end{tabular}

Table 2: Empirical power of different linearity tests at the nominal $5 \%$ significance level for $a=0.1$ and $b=0.1$. The DGP is given by equations in (1) and (3). Zhang is based on auxiliary regression (4), TI on (6) and Infeasible on (8).

All approaches lead to satisfying results in all considered scenarios. A direct comparison between the approach studied in Zhang (2012) and our suggested testing procedure (TI) shows that the latter one has higher power in most situations. Especially for the LSTAR case and in ESTAR models with a non-stationary inner regime $(\rho \geq 1)$ we observe substantial power gains. An exception is the case of ESTAR models with $(\rho=0)$, 
see Table 3. In this case the empirical power of both tests is very close to each other. Nonetheless, the higher $T$, the better performs the TI procedure.

ESTAR

\begin{tabular}{|c|c|c|c|c|c|c|c|c|}
\hline \multirow[b]{2}{*}{$\rho$} & \multirow[b]{2}{*}{$\phi$} & \multirow[b]{2}{*}{$\gamma$} & \multicolumn{3}{|c|}{$T=150$} & \multicolumn{3}{|c|}{$T=300$} \\
\hline & & & Zhang & $\mathrm{TI}$ & Infeasible & Zhang & $\mathrm{TI}$ & Infeasible \\
\hline \multirow[t]{10}{*}{0} & 0.7 & 0.1 & 15.0 & 18.9 & 19.6 & 31.4 & 36.1 & 36.2 \\
\hline & & 0.3 & 32.2 & 35.6 & 40.3 & 68.7 & 72.4 & 75.0 \\
\hline & & 0.5 & 25.5 & 24.7 & 30.5 & 57.2 & 59.3 & 62.3 \\
\hline & & 0.7 & 17.2 & 15.5 & 18.9 & 37.5 & 37.7 & 41.8 \\
\hline & & 0.9 & 10.9 & 9.6 & 12.8 & 23.4 & 24.5 & 27.5 \\
\hline & 0.9 & 0.1 & 23.5 & 27.7 & 29.2 & 52.9 & 59.2 & 60.4 \\
\hline & & 0.3 & 55.8 & 53.5 & 63.7 & 93.2 & 93.7 & 95.2 \\
\hline & & 0.5 & 33.1 & 25.3 & 38.0 & 66.5 & 60.5 & 69.8 \\
\hline & & 0.7 & 17.0 & 12.5 & 21.3 & 31.7 & 23.1 & 32.5 \\
\hline & & 0.9 & 10.3 & 7.5 & 13.6 & 16.3 & 12.2 & 17.0 \\
\hline \multirow[t]{10}{*}{1} & -0.7 & 0.1 & 46.3 & 52.4 & 63.4 & 81.8 & 86.0 & 90.4 \\
\hline & & 0.3 & 47.7 & 52.4 & 58.4 & 81.4 & 85.0 & 87.2 \\
\hline & & 0.5 & 36.9 & 39.9 & 45.3 & 68.1 & 72.6 & 74.6 \\
\hline & & 0.7 & 28.7 & 31.1 & 34.0 & 53.4 & 56.1 & 58.8 \\
\hline & & 0.9 & 19.3 & 21.4 & 23.5 & 40.3 & 43.0 & 44.4 \\
\hline & -0.9 & 0.1 & 58.4 & 65.2 & 72.6 & 91.6 & 94.3 & 96.3 \\
\hline & & 0.3 & 64.9 & 70.9 & 75.3 & 94.4 & 95.9 & 96.8 \\
\hline & & 0.5 & 58.5 & 61.5 & 65.4 & 87.9 & 90.7 & 91.8 \\
\hline & & 0.7 & 44.7 & 47.0 & 50.3 & 76.4 & 78.8 & 80.6 \\
\hline & & 0.9 & 35.5 & 38.8 & 40.3 & 63.5 & 66.3 & 67.8 \\
\hline \multirow[t]{10}{*}{1.5} & -1 & 0.1 & 74.8 & 76.6 & 100.0 & 90.7 & 91.9 & 100.0 \\
\hline & & 0.3 & 80.1 & 82.7 & 94.5 & 98.4 & 99.1 & 100.0 \\
\hline & & 0.5 & 62.5 & 66.6 & 79.1 & 94.0 & 95.1 & 97.8 \\
\hline & & 0.7 & 47.2 & 48.4 & 59.2 & 80.6 & 83.1 & 86.5 \\
\hline & & 0.9 & 35.4 & 36.5 & 41.3 & 64.6 & 67.1 & 71.7 \\
\hline & -1.4 & 0.1 & 93.6 & 94.6 & 100.0 & 99.9 & 99.8 & 100.0 \\
\hline & & 0.3 & 97.6 & 97.7 & 99.2 & 100.0 & 100.0 & 100.0 \\
\hline & & 0.5 & 92.4 & 93.9 & 96.3 & 100.0 & 99.9 & 100.0 \\
\hline & & 0.7 & 83.5 & 85.8 & 88.7 & 99.0 & 99.1 & 99.1 \\
\hline & & 0.9 & 70.0 & 70.5 & 73.4 & 94.6 & 94.6 & 95.7 \\
\hline
\end{tabular}

Table 3: Empirical power of different linearity tests at the nominal $5 \%$ significance level for $a=0$ and $b=0$. The DGP is given by equations in (1) and (2). Zhang is based on auxiliary regression (4), TI on (5) and Infeasible on (7). 
ESTAR

\begin{tabular}{|c|c|c|c|c|c|c|c|c|}
\hline \multirow[b]{2}{*}{$\rho$} & \multirow[b]{2}{*}{$\phi$} & \multirow[b]{2}{*}{$\gamma$} & \multicolumn{3}{|c|}{$T=150$} & \multicolumn{3}{|c|}{$T=300$} \\
\hline & & & Zhang & TI & Infeasible & Zhang & TI & Infeasible \\
\hline \multirow[t]{10}{*}{0} & 0.7 & 0.1 & 14.3 & 17.2 & 18.2 & 29.1 & 34.6 & 34.9 \\
\hline & & 0.3 & 30.5 & 33.4 & 37.1 & 66.4 & 71.0 & 73.3 \\
\hline & & 0.5 & 24.3 & 22.7 & 28.2 & 58.3 & 59.4 & 62.6 \\
\hline & & 0.7 & 18.0 & 15.1 & 18.7 & 36.8 & 37.4 & 42.6 \\
\hline & & 0.9 & 11.0 & 10.0 & 11.8 & 23.6 & 22.9 & 26.7 \\
\hline & 0.9 & 0.1 & 22.7 & 28.7 & 30.4 & 51.6 & 57.1 & 57.6 \\
\hline & & 0.3 & 58.2 & 55.1 & 63.5 & 92.4 & 93.0 & 94.7 \\
\hline & & 0.5 & 35.1 & 25.5 & 38.8 & 66.6 & 60.8 & 69.8 \\
\hline & & 0.7 & 17.4 & 12.2 & 21.2 & 32.3 & 24.4 & 31.5 \\
\hline & & 0.9 & 9.9 & 6.8 & 14.4 & 16.8 & 11.6 & 17.5 \\
\hline \multirow[t]{10}{*}{1} & -0.7 & 0.1 & 45.7 & 51.5 & 60.9 & 82.9 & 87.0 & 91.1 \\
\hline & & 0.3 & 46.0 & 49.9 & 55.6 & 81.6 & 84.3 & 87.2 \\
\hline & & 0.5 & 38.0 & 41.4 & 45.2 & 67.6 & 71.4 & 73.7 \\
\hline & & 0.7 & 27.4 & 31.0 & 33.1 & 52.0 & 56.2 & 58.3 \\
\hline & & 0.9 & 22.0 & 23.8 & 24.8 & 42.9 & 45.6 & 47.4 \\
\hline & -0.9 & 0.1 & 60.6 & 65.7 & 75.3 & 91.3 & 94.3 & 95.9 \\
\hline & & 0.3 & 66.8 & 71.3 & 76.6 & 94.2 & 95.8 & 96.7 \\
\hline & & 0.5 & 58.4 & 60.5 & 64.0 & 87.0 & 89.1 & 90.4 \\
\hline & & 0.7 & 43.9 & 47.1 & 50.1 & 76.5 & 79.8 & 80.5 \\
\hline & & 0.9 & 33.5 & 36.0 & 38.1 & 62.2 & 64.1 & 66.1 \\
\hline \multirow[t]{10}{*}{1.5} & -1 & 0.1 & 74.0 & 75.7 & 100.0 & 91.3 & 91.3 & 100.0 \\
\hline & & 0.3 & 80.1 & 82.8 & 94.7 & 98.2 & 98.6 & 100.0 \\
\hline & & 0.5 & 63.1 & 66.4 & 77.4 & 93.0 & 93.3 & 96.8 \\
\hline & & 0.7 & 47.4 & 49.2 & 58.1 & 81.1 & 83.1 & 86.8 \\
\hline & & 0.9 & 35.8 & 38.0 & 45.0 & 64.4 & 67.9 & 71.0 \\
\hline & -1.4 & 0.1 & 93.6 & 94.1 & 100.0 & 99.7 & 99.8 & 100.0 \\
\hline & & 0.3 & 96.9 & 97.7 & 99.7 & 100.0 & 100.0 & 100.0 \\
\hline & & 0.5 & 92.5 & 92.5 & 95.4 & 100.0 & 100.0 & 100.0 \\
\hline & & 0.7 & 82.4 & 83.3 & 86.6 & 98.6 & 98.8 & 98.9 \\
\hline & & 0.9 & 70.3 & 71.2 & 75.0 & 95.3 & 95.0 & 96.0 \\
\hline
\end{tabular}

Table 4: Empirical power of different linearity tests at the nominal $5 \%$ significance level for $a=0.1$ and $b=0.1$. The DGP is given by equations in (1) and (2). Zhang is based on auxiliary regression (4), TI on (5) and Infeasible on (7).

The power difference between the TI procedure and the infeasible benchmark is below $10 \%$ for most of the cases of the evaluated scenarios. Especially the ESTAR parametriza- 
Real world parameter settings for ESTAR models applied to real exchange rates

\begin{tabular}{|c|c|c|c|c|c|c|c|c|c|}
\hline \multirow[b]{2}{*}{$\gamma$} & \multirow[b]{2}{*}{$\sigma$} & \multirow[b]{2}{*}{$a$} & \multirow[b]{2}{*}{$b$} & \multicolumn{3}{|c|}{$T=150$} & \multicolumn{3}{|c|}{$T=300$} \\
\hline & & & & Zhang & $\mathrm{TI}$ & Infeasible & Zhang & TI & Infeasible \\
\hline $0.294 \quad-0.049$ & 0.033 & 0 & 0 & 6.0 & 8.3 & 21.8 & 7.6 & 11.5 & 24.9 \\
\hline \multirow[t]{3}{*}{$(\mathrm{USD} / \mathrm{FF})$} & & 0 & 0.1 & 5.7 & 9.3 & 24.0 & 7.1 & 11.4 & 26.1 \\
\hline & & 0.1 & 0 & 6.0 & 8.0 & 23.0 & 8.6 & 11.9 & 26.6 \\
\hline & & 0.1 & 0.1 & 5.5 & 8.7 & 22.2 & 7.7 & 11.0 & 26.7 \\
\hline 0.257 & 0.034 & 0 & 0 & 4.5 & 7.4 & 22.4 & 7.2 & 10.9 & 25.6 \\
\hline \multirow[t]{3}{*}{$(\mathrm{USD} / \mathrm{DM})$} & & 0 & 0.1 & 6.1 & 8.5 & 20.7 & 6.6 & 10.1 & 26.8 \\
\hline & & 0.1 & 0 & 5.3 & 8.1 & 22.8 & 7.8 & 12.0 & 27.4 \\
\hline & & 0.1 & 0.1 & 5.8 & 8.2 & 21.3 & 7.5 & 10.5 & 26.8 \\
\hline $0.452 \quad-0.149$ & 0.033 & 0 & 0 & 4.7 & 7.6 & 22.4 & 7.7 & 12.6 & 36.0 \\
\hline \multirow[t]{3}{*}{ (USD/GBP) } & & 0 & 0.1 & 6.3 & 8.9 & 23.0 & 8.6 & 12.6 & 36.5 \\
\hline & & 0.1 & 0 & 4.6 & 7.6 & 22.9 & 8.4 & 13.2 & 38.9 \\
\hline & & 0.1 & 0.1 & 6.2 & 9.2 & 23.6 & 9.2 & 12.8 & 35.6 \\
\hline $0.170 \quad-0.516$ & 0.034 & 0 & 0 & 6.0 & 8.3 & 33.1 & 6.4 & 9.3 & 36.7 \\
\hline \multirow[t]{3}{*}{ (USD/JPY) } & & 0 & 0.1 & 5.0 & 6.6 & 34.8 & 7.9 & 10.2 & 37.4 \\
\hline & & 0.1 & 0 & 3.9 & 7.5 & 35.0 & 8.3 & 10.5 & 38.4 \\
\hline & & 0.1 & 0.1 & 4.8 & 7.2 & 35.2 & 7.7 & 10.5 & 37.3 \\
\hline
\end{tabular}

Table 5: Empirical power of different linearity tests at the nominal $5 \%$ significance level for real world parameter settings taken from Taylor et al. (2001) (see their Table 3). Note that $\rho=1$ and $\phi=-1$. The standard deviation of $\varepsilon_{t}$ is denoted as $\sigma$. The currencies are USD: US Dollar, FF: French Franc, DM: Deutsche Mark and JPY: Japanese Yen.

tion with $\{\rho=1.5, \phi=-1\}$ has a relatively high difference if $T=150$ and the non-stationary inner interval of the process is large. For $T=300$ the difference decreases and is above the $10 \%$ level for the LSTAR parametrization $\{\rho=0, \phi=0.7, \gamma=0.3\}$ only. When considering the presence of a deterministic trend $(b \neq 0)$, we find no substantial differences in the results demonstrating the invariance of the tests to a linear trend in the DGP.

Next, we compare some of our results with the results for the $W_{T}^{0}$ test in Harvey and Leybourne (2007). Their $W_{T}^{0}$ test is based on the auxiliary regression

$$
y_{t}=\beta_{0}+\beta_{1} y_{t-1}+\beta_{2} y_{t-1}^{2}+\beta_{3} y_{t-1}^{3}+u_{t}
$$

and does not account for linear deterministic trends. In order to ensure a meaningful comparison, we compare the results from our Tables 1 and 3 (with $b=0$, i.e. no deterministic trend is present in the DGP) to the one reported in Harvey and Leybourne (2007) (their Table 3). ${ }^{3}$ By construction, it is expected that the $W_{T}^{0}$ test outperforms the

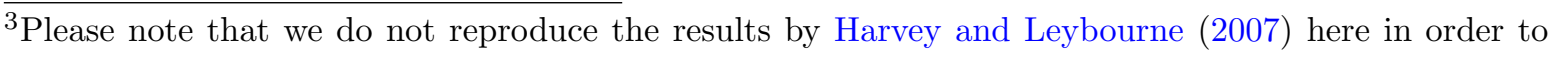
save space. 
TI procedure for ESTAR models. Indeed, this result is confirmed and can be explained by the fact that the TI procedure unnecessarily accounts for a deterministic trend which is actually not present in the DGP. Interestingly, we observe for LSTAR models that the TI procedure always outperforms the $W_{T}^{0}$ test. Notably, the power loss induced by falsely accounting for a linear trend term is overcompensated by using a parsimonious test regression, where only the second power of $y_{t-1}$ is used (compare equation (9) to (6)).

Finally, we consider real world parameter settings. ${ }^{4}$ The following analysis is based on estimation results for real exchange rate data reported in Taylor, Peel, and Sarno (2001). We simulate highly persistent, but mean-reverting ESTAR models with and without linear deterministic trends for four major real bilateral dollar exchange rates. The simulation results are reported in Table 5. The following conclusions can be drawn: (i) it is difficult for any test to detect nonlinearity as the power is very low; (ii) the power gap between the infeasible test and the other two tests is moderate; (iii) the empirical power of the infeasible test itself does not exceed $40 \%$ suggesting difficulties in rejecting the false null hypothesis of linearity. Lastly, we observe that (iv) the presence of a linear deterministic trend does not alter the previous conclusions and moreover, that (v) the TI procedure dominates the one used by Zhang, even though the power gains may be small in most settings.

When comparing our findings to the one in Taylor et al. (2001) who study the empirical power of linear unit root tests against nonlinear mean-reverting ESTAR models by considering the same parameter estimates, striking similarities appear. Taylor et al. (2001) also demonstrate difficulties to reject the false null of linearity in practice. Potential explanations for some of these features are discussed in Kruse, Frömmel, Menkhoff, and Sibbertsen (2012). However, as shown in Taylor et al. (2001), power gains can be made when using panel unit root tests instead of univariate ones. We conjecture that a similar result can be found in our case as well.

\section{Conclusion}

This article reconsiders linearity testing against STAR models when deterministic trends might be present in the data. In contrast to Zhang (2012), we conclude that testing procedures based on Teräsvirta (1994) work well in most situations. Moreover, we find that a slight modification of the auxiliary test regression leads often to higher empirical power. This conclusion does not change when real world parameter settings obtained

\footnotetext{
${ }^{4}$ We are grateful to an anonymous referee for bringing this issue to our attention.
} 
for real exchange rate data are considered.

\section{References}

Cuestas, J. C. And D. Garratt (2011): "Is real GDP per capita a stationary process? Smooth transitions, nonlinear trends and unit root testing," Empirical Economics, 41, 555-563.

Harvey, D. I. And S. J. Leybourne (2007): "Testing for time series linearity," Econometrics Journal, 10, 149-165.

KILIÇ, R. (2004): "Linearity tests and stationarity," Econometrics Journal, 7, 55-62.

Kruse, R., M. Frömmel, L. Menkhoff, and P. Sibbertsen (2012): "What do we know about real exchange nonlinearities?" Empirical Economics, forthcoming.

SANDBERG, R. (2008): "Critical values for linearity tests in time-varying smooth transition autoregressive models when data are highly persistent," Econometrics Journal, $11,638-647$.

Taylor, M. P., D. A. Peel, And L. Sarno (2001): "Nonlinear mean-reversion in real exchange rates: Toward a solution to the purchasing power parity puzzles," International Economic Review, 42, 1015-1042.

Teräsvirta, T. (1994): "Specification, Estimation, and Evaluation of Smooth Transition Autoregressive Models," Journal of the American Statistical Association, 89, $208-218$.

ZHANG, L. (2012): "Test for linearity against STAR models with deterministic trends," Economics Letters, 115, 16-19. 